\title{
An Efficient Mathematical Approach for an Indoor Robot Localization System
}

\author{
Israa Sabri A. AL-Forati \\ Electrical Engineering Department \\ University of Basrah \\ Basrah Iraq \\ israa.subri.1@email.com
}

\author{
Abdulmuttalib Rashid \\ Electrical Engineering Department \\ University of Basrah \\ Basrah Iraq \\ abdturky@email.com
}

\author{
Fatemah Al-Assfor \\ Computer Engineering Department \\ University of Basrah \\ Basrah Iraq \\ fatimah_alasfoor@yahoo.com
}

Abstract-In a counterfeit clever control procedure, another productive methodology for an indoor robot localization framework is arranged. In this paper, a new mathematic calculation for the robot confinement framework utilizing light sensors is proposed. This procedure takes care of the issue of localization (position recognizing) when utilizing a grid of LEDs distributed uniformly in the environment, and a multiportable robot outfitted with a multi-LDRs sensor and just two of them activate the visibility robot. The proposed method is utilized to assess the robot's situation by drawing two virtual circles for each two LDR sensors; one of them is valid and the other is disregarded according to several suggested equations. The midpoint of this circle is assumed to be the robot focus. The new framework is simulated on a domain with $(n * n)$ LEDs exhibit. The simulation impact of this framework shows great execution in the localization procedure.

Index Terms-Localization system, LDR sensors, LEDs array

\section{INTRODUCTION}

Previously, the localization of an indoor robot was a difficult mission due to many physical restrictions. In the indoor robot field, the robot localization systems and formation had been the specific interest [1]. Robot teams can be used for many tasks, such as surveillance, inspection and for the formation. In these circumstances, robots can be required to improve the path planning or to navigate in the formation [2-7]. The most wellknown localization approach is the Global Positioning System (GPS), but there are some limitations, such as its power consumption, high cost and large size [8], thus making this approach unusable. Furthermore, the GPS cannot be used in indoor environments. This caused an increasing interest in other procedures that deal with the requirements of indoor localization [9, 10]. A Grid of Wireless Sensors contains many nodes of sensor gap separators to cooperatively display environmental conditions and offers different advantages, such as object tracking, traffic monitoring, navigating ships, volcanic eruptions, and calculating radiation planes from nuclear reactors [11]. The robot's position is estimated by dividing the labeled sensors into clusters and then the middle of each cluster denotes the location of the robot [12]. The sensor is a small tool that is used to measure or sense a quantity, then translates it over a relationship to a new motion that is observed and exhibited or for extra processing. Moreover, they are used for many kinds of measurements, for example: sound, distance, motion, temperature, pressure, and light [13,14]. As a fast increase in smart skill, we are looking for a sensor that offers accurate distance detection and isn't too costly. Various techniques are used for indoor localization, such as the scheme which is based on radio frequency identification RFID, ultrawideband, infrared, Bluetooth, ultrasound, Wi-Fi, and infrared $[15,16]$. More precisely, the present work can be presented with the following points: The localization problem is solved by using an array of sensors (of different types and dimensions) distributed in the environment at regular intervals. The essential information about the localization procedure is collected by scanning the array's sensors in the environment. The scanning progression is done in order to identify the active sensors in the environment, which receive signals from the sensor that the robot is equipped with. The problem appears when only two sensors detect the availability of the robot. However, it doesn't matter 
when more than two sensors are detected due to the requirements needed to draw a circle. Here, a new, efficient equation is proposed to solve the problem. The robot's position is estimated to be in the middle of the virtual circle. This paper aims to solve the problem of localization for an indoor robot when only two sensors are in the environment detecting the availability of the robot. The solution to this problem has been found by using an efficient equation technique for various situations and by providing accurate calculations to sidestep any mistake that may be faced while determining the position of the robots with different condition locations.

\section{RELATED WORK}

The localization field was practically advanced by proposing a simple indoor position system (IPS) using different technologies [17] like Bluetooth ultrasound, vision analysis (WLAN), (IR) sensors TX \& RX sensor networks, radio-frequency identification (RFID), magnetic signals and audible sound, and ultra-wideband (UWB). These techniques extended to the IPS scope in universities, companies, and research areas ,But these systems have Constraints such as the need for additional infrastructure, Low resolution, noise, electromagnetic interference, low security multipath effects, , etc. [18] Normally much research has been directed as of late on utilizing these indoor LED lighting and VLC procedures as an option indoor positioning signal for spots where satellite worldwide situating framework (GPS) signals are not accessible [9]. The greatest favorable position of utilizing VLC strategies for indoor localization is that no extra framework is required for the establishment since the framework uses an existing foundation of LED lights sensor, which lessens the expense of the ILS.In the 1990s, the primary indoor localization at T\&AT Cambridge was a system where it was fitted by using symbolic location information to each active badge, such as the position in a building and the site where the active badge is. The problem was solved by using optical filters and many electronics to diffuse the trouble from the sources itself and by using noise cancelling algorithms at the receivers in order to accomplish the signal processing, though it increased the cost of the localization system [20].To demonstrate the effectiveness of the proposed system simulation of an indoor localization system using LED array of $(n * n)$ sensors, and a mobile robot equipped with LDRs sensor as a receiver.

\section{III.THE LOCALIZATION SYSTEM}

This work presents a new mathematical algorithm for the indoor robot localization system using light sensors. The sensors are separated into two classes: the first one is characterized by eight light dependent resistors (LDR) close-fitting onto the robot, and the other one is categorized by an array of LEDs distributed at regular intervals in the environment $(64 * 64),(32 * 32)$ and $(16 * 16)$ LEDs. Fig. 1 shows an environment with a (16*16) LED matrix. The central unit exanimates the LED array to distinguish the LDR sensors in its sensing range. Only LEDs within the sensing range of the LDRs are identified. In order to estimate the robot's position, for a number of receivers (LDRs), the minimum bounded circle algorithm is used to draw a minimum circle that contains all of the points; as a result, the center of this circle is assumed to be the robot's position. At least three of the LDR sensors locations can be used to draw a circle, with the center representing the location of the robot. The problem occurs when only two LDR sensors are identified. This paper suggests a mathematical approach for robot localization with only two LDR sensors.

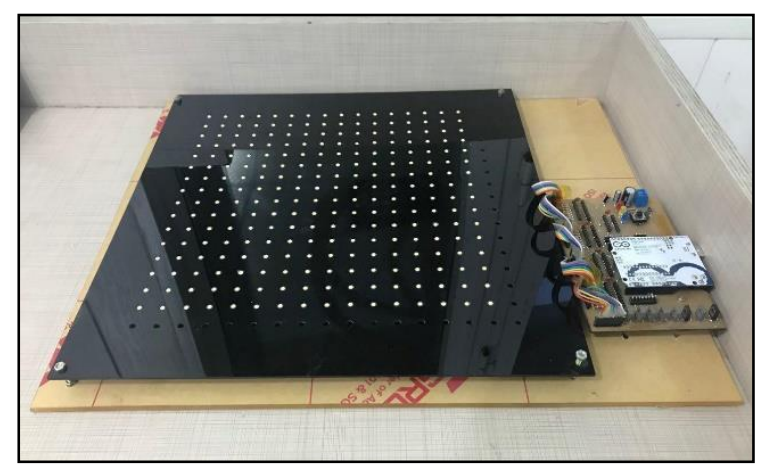

Fig. $1(16 * 16)$ LED matrix environment with central unit.

\section{The LEDs sensors matrix}

The planned indoor environment involves a $2 \mathrm{D}$ environment with many holes distributed regularly and filled with a matrix of LEDs sensors. The proposed localization system involves 4-robots, as shown in Fig.2., and 8 LDRs fixed on each robot, as shown in Fig.3.The schematic diagram for each robot is shown in Fig.4.The robot is shown in Fig.5. The prepared axis of each LED sensor is assumed to be known. 


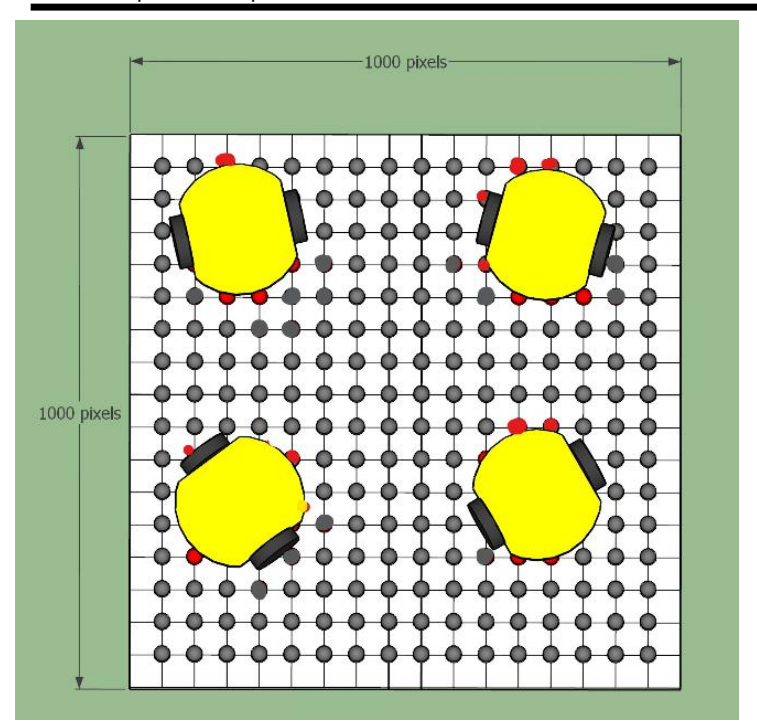

Fig. 2 The indoor localization environment. ( (16*16) LEDs array environment with 4 robots.

The four robots' localization procedure is complete by scanning the matrix of LEDs to identify which LDR sensors are detecting the light of the LEDs. The sensed LEDs are characterized one by one and identified by a red color, as shown in Fig. 2.

\section{THE MATHEMATICAL MODELS}

After detecting the LEDs light by using an LDR sensor, we have a problem if only two LDRs are recognized because it cannot draw a circle using only two points; for every two points there is a possibility to estimate two robot positions. In this section, a new mathematical equation model is proposed in order to solve the previous problem. Ten cases are planned in order to gather the overall conditions to estimate the robot's location. In each case, two locations $((\mathrm{x} 1, \mathrm{y} 1)$ and $(\mathrm{x} 2, \mathrm{y} 2))$ are used to estimate the robot's position.

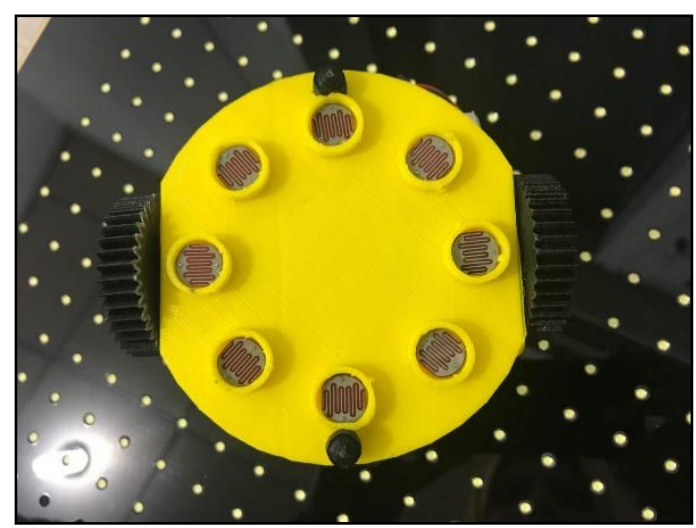

Fig. 3 eight LDRs fixed on the lower side of each robot.

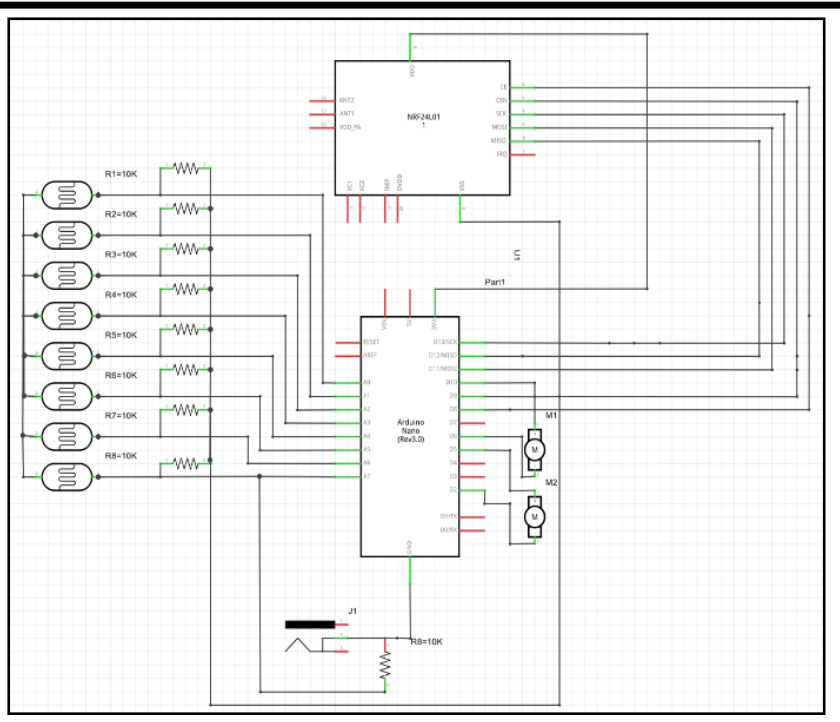

Fig. 4 the schematic diagram for each robot.

Two locations ((xc, yc) and $(\mathrm{x} 3, \mathrm{y} 3))$ are suggested to represent the centers of two circles with equal radiuses. According to the suitable case, the point (xc, yc) is chosen as the center of the robot and the other point $(x 3, y 3)$ is omitted. Fig. 6 shows the environment used to discuss the ten cases, which shows the distribution of the eight LDR sensors on the lower side of the robot. The cases are obtained from dividing the environment into four quarters and the name of each case dependent on the location of the active LDR sensor. The LDR sensor with a high $\mathrm{X}$ axis always represents the first active sensor $(\mathrm{x} 1, \mathrm{y} 1)$.

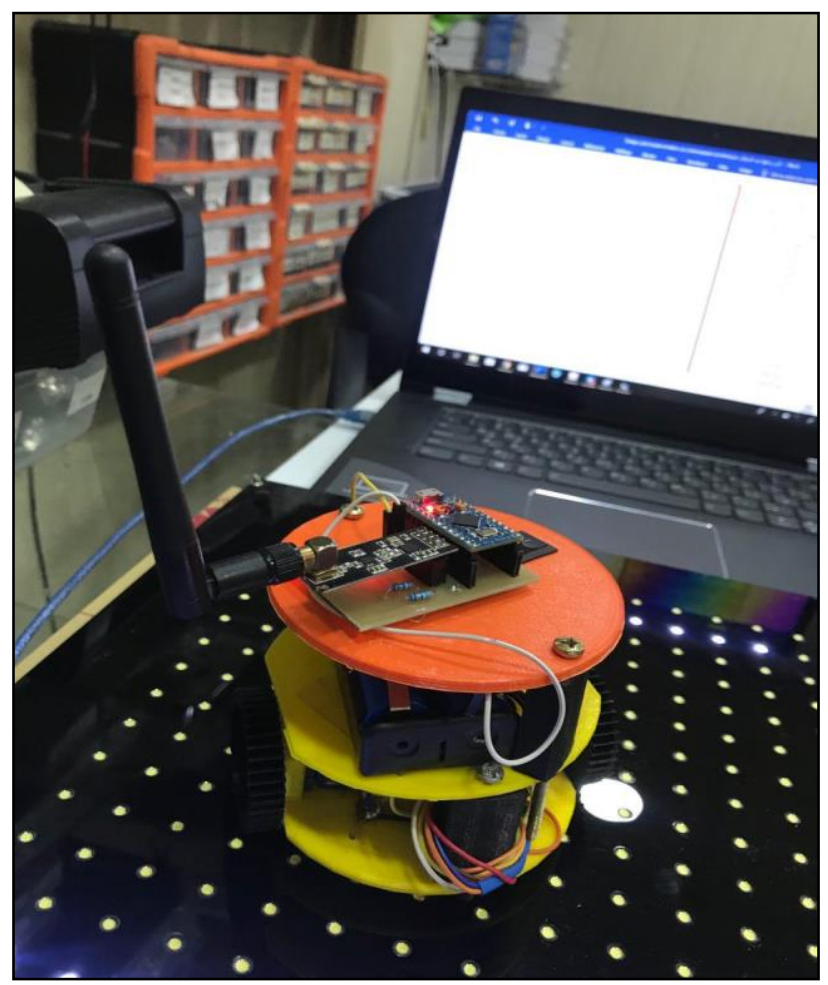

Fig. 5 The robot with eight LDR sensors. 


\section{2) FIRST- SECOND QUARTERS CASE:}

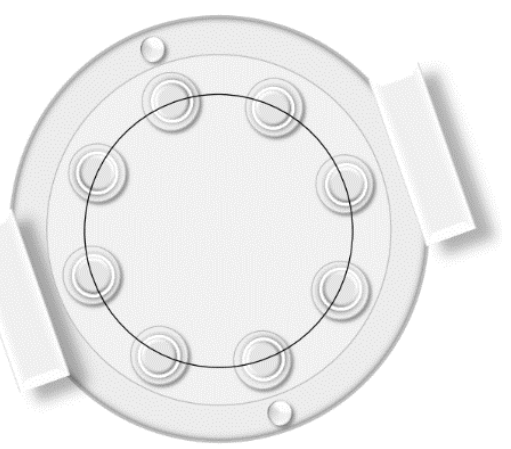

Fig. 6 The lower side of the robot with eight LDR sensors.

\section{1) FIRST- FIRST QUARTERS CASE:}

This case represents both of the active LDR sensors that are located in a first quadrature, as shown in Fig. 7. The coordinate axis of the robot is computed according to the following equation:

$$
\begin{aligned}
& \theta_{3}=\tan ^{-1} \frac{(Y 2-Y 1)}{(X 2-X 1)} \\
& \theta_{2}=\left(180-\theta_{1}\right) / 2 \\
& \theta_{4}=\theta_{2}+\theta_{3} \\
& X c=X 1-R \cos \theta_{4} \\
& Y c=Y 1-R \sin \theta_{4}
\end{aligned}
$$

Where $(\mathrm{Xc}, \mathrm{Yc})$ is the coordinate axis of the robot and $\mathrm{R}$ is the distance between the center of the robot and the LDR sensors.

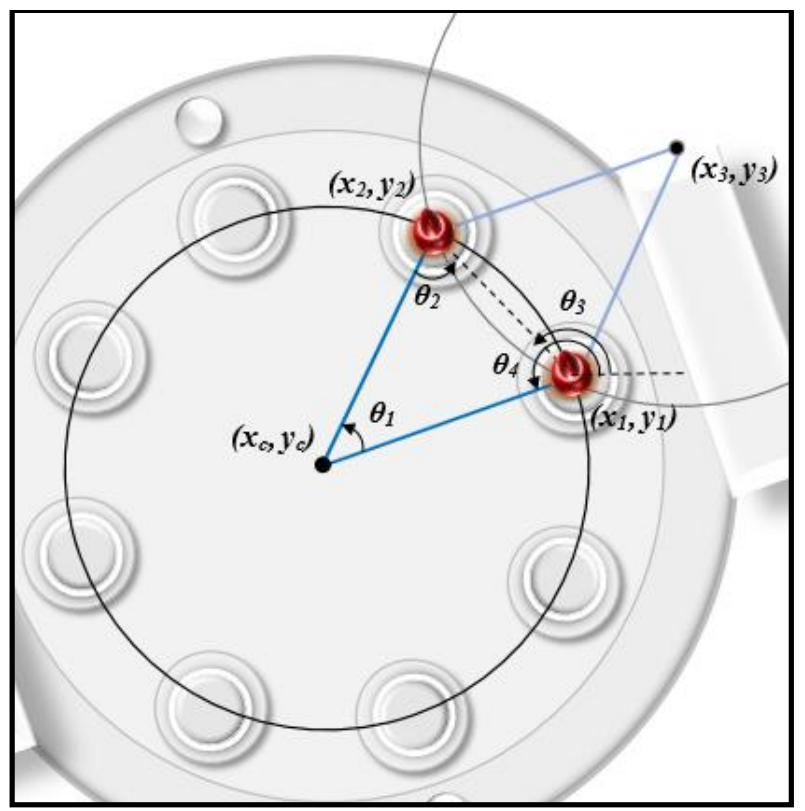

Fig. 7 The first-first quarters case
This case represents the first active LDR sensor, which is located in the first quadrature, and the second active LDR sensor, which is located in the second quarter, as shown in Fig. 8. The coordinate axis of the robot is computed according to the same equations used in the last case.

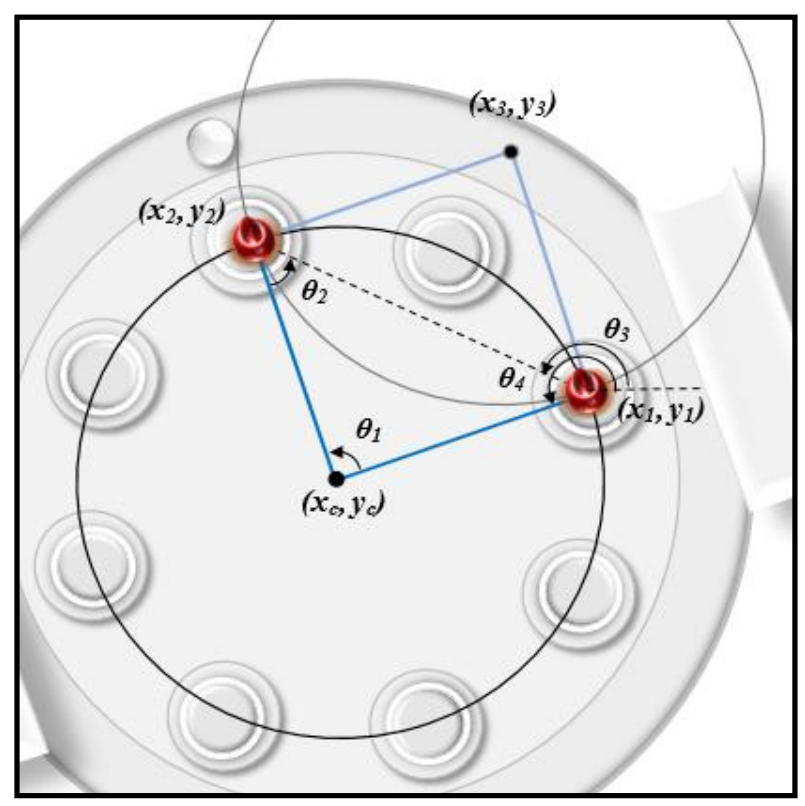

Fig. 8 The first-second quarters case.

\section{3) FIRST- THIRD QUARTERS CASE:}

This case represents the first active LDR sensor, which is located in the first quadrature, and the second active LDR sensor, which is located in the third quarter, as shown in Fig.9.

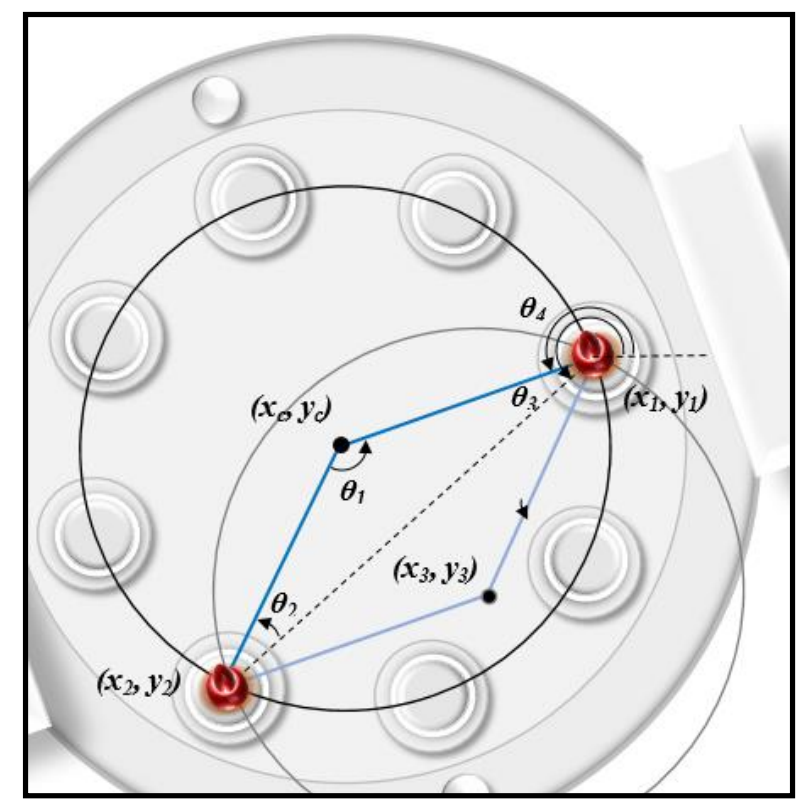

Fig. 9 The first-third quarters case. 
The coordinate axis of the robot is computed according to the following equations: Use (1) and (2) to compute $\theta 3$ and $\theta 2$

$$
\theta_{4}=\theta_{3}-\theta_{2}
$$

Use (5) and (6) to compute the coordinate axis of the robot.

\section{4) FIRST- FOURTH QUARTERS CASE:}

This case represents the first active LDR sensor, which is located in the first quadrature, and the second active LDR sensor, which is located in the fourth quarter, as shown in Fig. 10. The coordinate axis of the robot is computed according to the following equations:

Use (1), (2) and (6) to compute $\theta 3, \theta 2$ and $\theta 4$, respectively. The coordinate axis of the robot is computed by using (4) and (5).

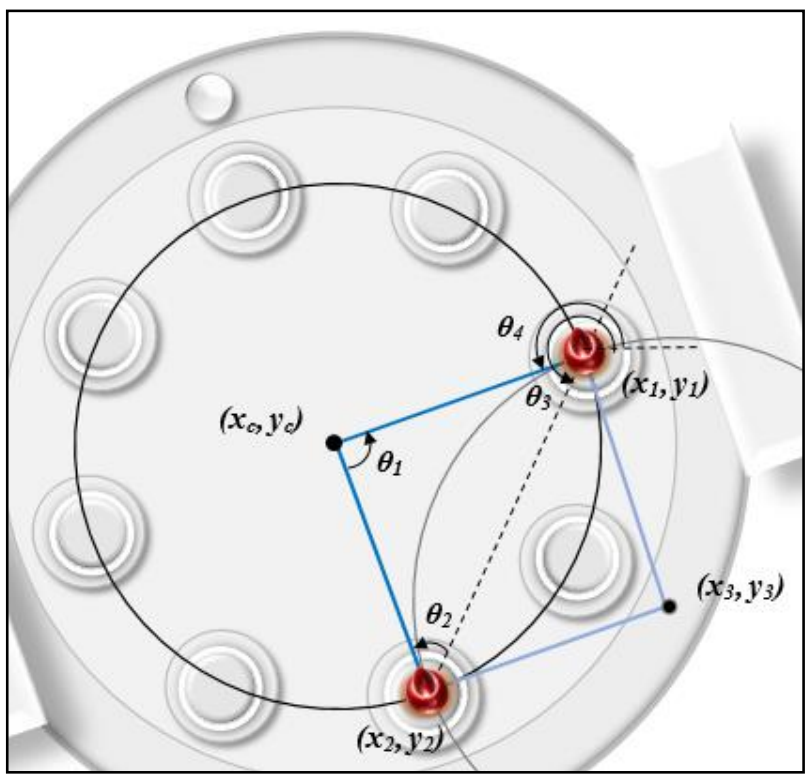

Fig. 10 The first-fourth quarters case.

\section{5) SECOND- SECOND QUARTERS CASE:}

This case represents that both the first and the second active LDR sensors are located in the second quadrature of the environment, as shown in Fig. 11. The coordinate axis of the robot is computed according to the following equations:

Use (1), (2) and (3) to compute $\theta 3, \theta 2$ and $\theta 4$, respectively. The coordinate axis of the robot is computed by using (7). The y axis is computed by using (5).

$$
X c=X 1+R \cos \theta_{4}
$$

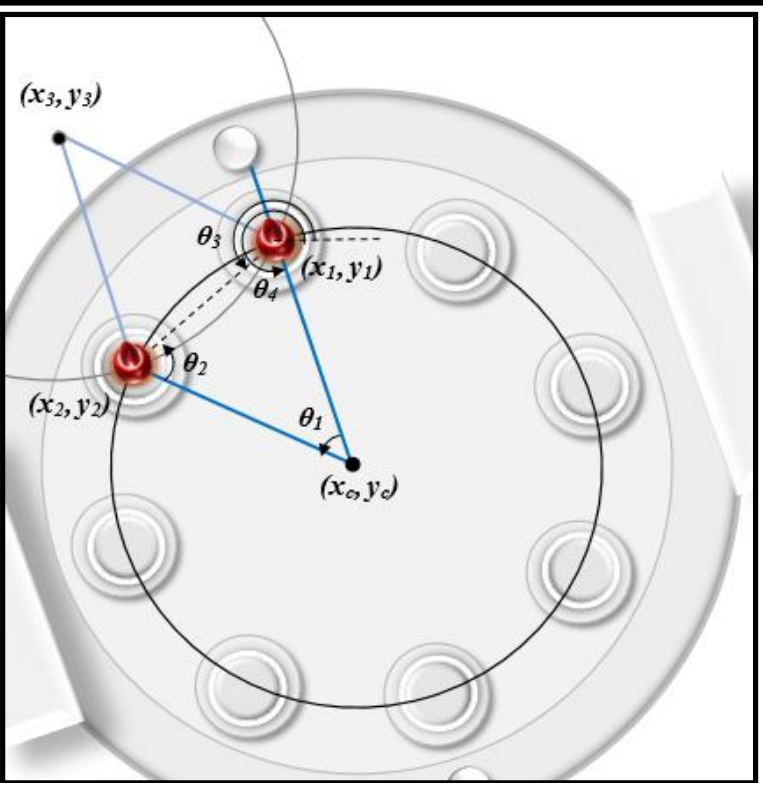

Fig. 11 The second-second quarters case.

\section{6) SECOND- THIRD QUARTERS CASE:}

This case represents the first active LDR sensor, which is located in second quadrature, and the second active LDR sensor, which is located in the third quarter, as shown in Fig. 12. The coordinate axis of the robot is computed according to the following equations: Use (1), (2) and (3) to compute $\theta 3, \theta 2$ and $\theta 4$, respectively. The coordinate axis of the robot is computed by using (5) and (7).

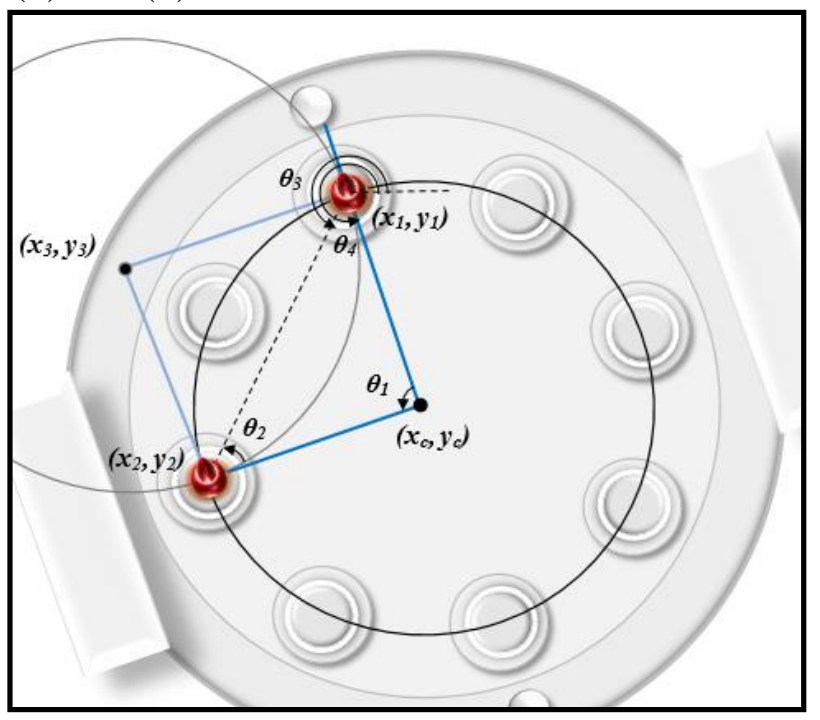

Fig. 12 The second-third quarters case.

7) SECOND- FOURTH QUARTERS CASE:

This case represents the first active LDR sensor. which is located in fourth quadrature (with maximum $\mathrm{X}$ axis), and the second active LDR sensor, which is located in the second quarter, as shown in Fig.13. 


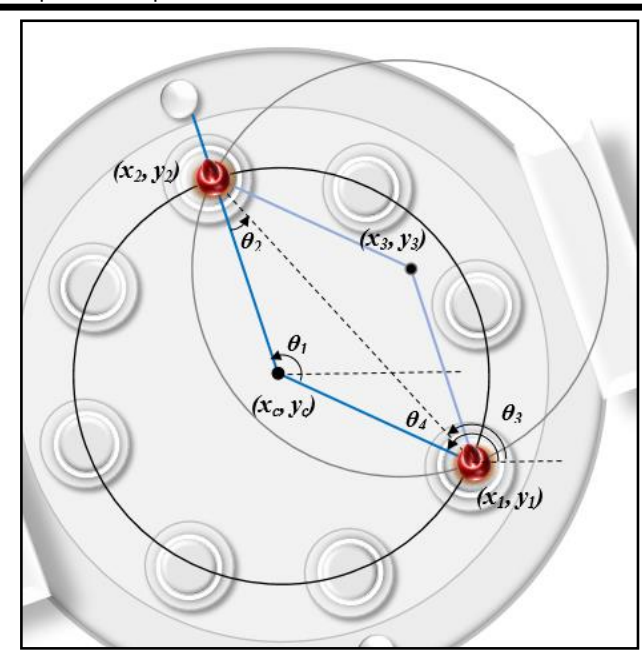

Fig. 13 The second-fourth quarters case.

The coordinate axis of the robot is computed according to the following equations:

Use (1), (2) and (3) to compute $\theta 3, \theta 2$ and $\theta 4$, respectively. The coordinate $\mathrm{X}$ axis of the robot is computed by using (4). The $\mathrm{Y}$ axis is computed by using (8).

$$
Y_{c}=Y 1+R \sin \theta_{4}
$$

\section{8) THIRD- THIRD QUARTERS CASE:}

This case represents both the first and the second active LDR sensors, which are located in third quadrature of the environment, as shown in Fig. 14. The coordinate axis of the robot is computed according to the following equations:

Use (1), (2) and (6) to compute $\theta 3, \theta 2$ and $\theta 4$, respectively. The coordinate axis of the robot is computed by using (5) and (7).

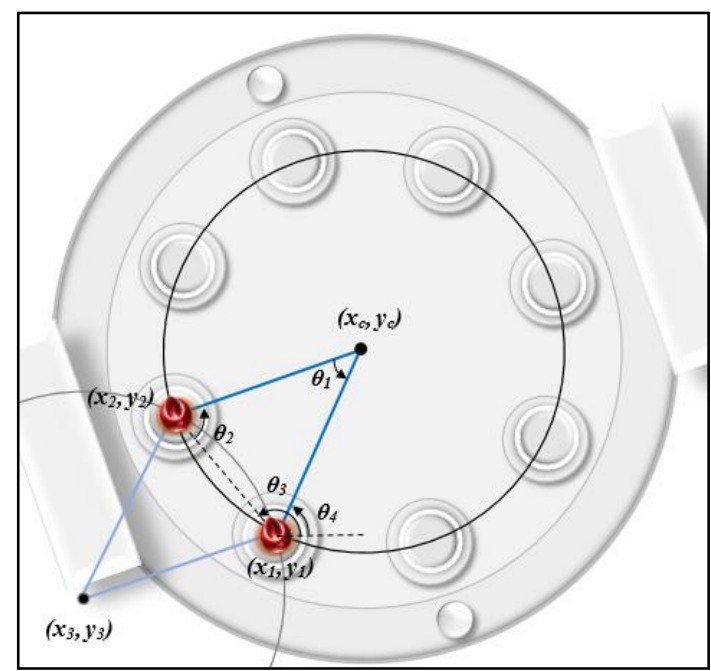

Fig. 14 The third-third quarters case.
9) THIRD- FOURTH QUARTERS CASE:

This case represents the first active LDR sensor. which is located in fourth quadrature (with maximum $X$ axis), and the second active LDR sensor, which is located in the third quarter, as shown in Fig.15. The coordinate axis of the robot is computed according to the following equations: Use (1), (2) and (6) to compute $\theta 3, \theta 2$ and $\theta 4$, respectively. The coordinate axis of the robot is computed by using (4) and (7).

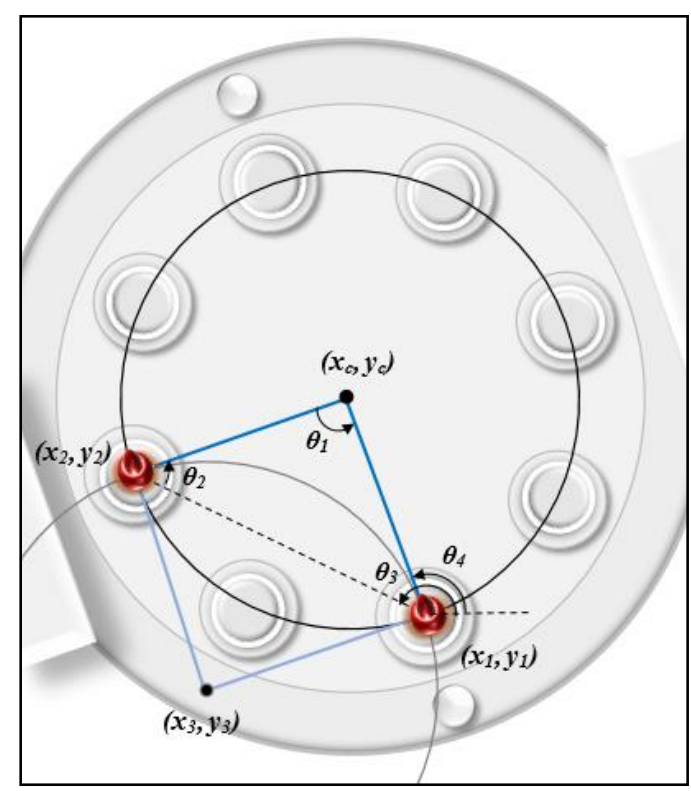

Fig. 15 The third-fourth quarters case.

10) FOURTH- FOURTH QUARTERS CASE:

This case represents both the first and second active LDR sensors, which are located in fourth quadrature of the environment (Fig. 16).

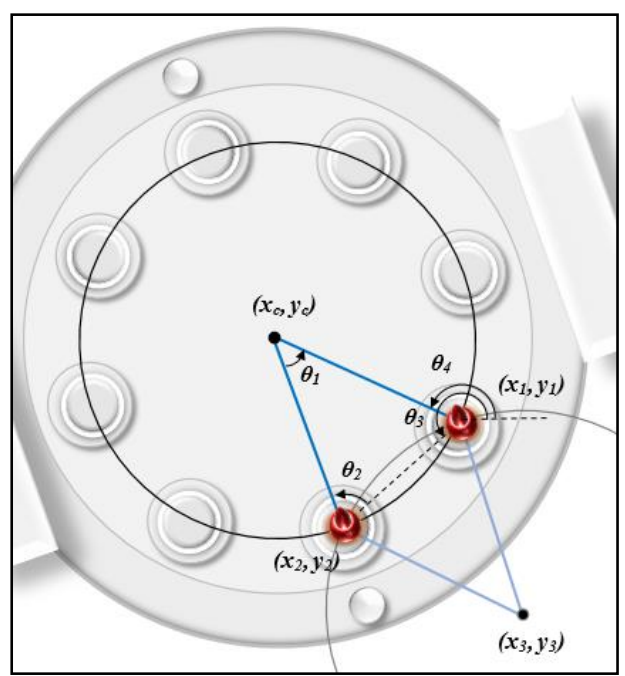

Fig. 16 The fourth-fourth quarters case. 
Coordinate axis of the robot is computed according to the following equations:

Use (1), (2) and (6) to compute $\theta 3, \theta 2$ and $\theta 4$, respectively. The coordinate axis of the robot is computed by using (4) and (7).

\section{SIMULATION RESULTS}

This paper suggests a new mathematic algorithm for the robot localization system using light sensors. The simulations are implemented on a robot with eight LDR sensors and they are repeated for 40 different topologies with different LDR sensing ranges, different robot locations, and different distances between the LDR sensors. $32 * 32$ LEDs matrix are distributed uniformly in a 1000*1000 Pixels environment. The system parameters used in these simulations are:

\section{A. LDRs diameter sensors equipped on each robot.}

\section{B. Maximum sensing range of the LDR sensor.}

Figs. 17, 18 and 19 show the localization simulations on a robot with two LDR sensors.
The first simulation is implemented on a robot with two LDR sensors with diameters equal to 20, 60 and 100 Pixels and is repeated for different sensing ranges of this sensor. Fig. 20 shows the simulation result obtained by repeating the first simulation 40 times with random locations of the robot to obtain the relationship between the percentage of the full sensing range and the different values of the sensing range.

The result shows that the best sensing range to obtain a full sensing range is 40 Pixels.

The second simulation is implemented for comparison on the average of the LDR sensor error occurring on robots that have two LDR sensors. These comparisons are tested in the localization process for robots with different sensing ranges. Fig. 21 shows a comparison of the results when the sensing range of each LDR is 20, 60 and 100 Pixels. This comparison shows that the average error is reduced as the distance between LDRs are also reduced.

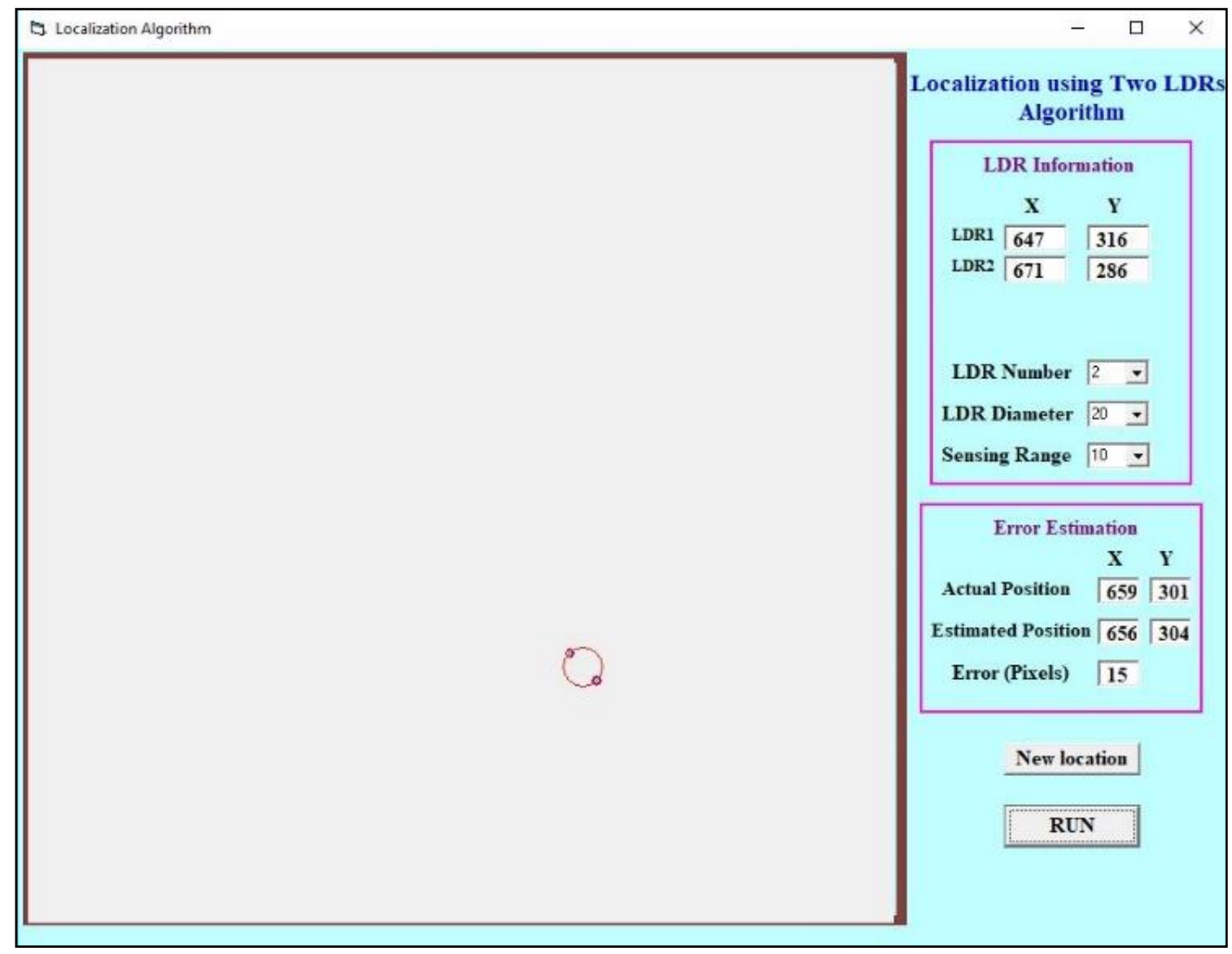

Fig. 17 Simulation for robot localization with a 20 Pixel LDR diameter. 


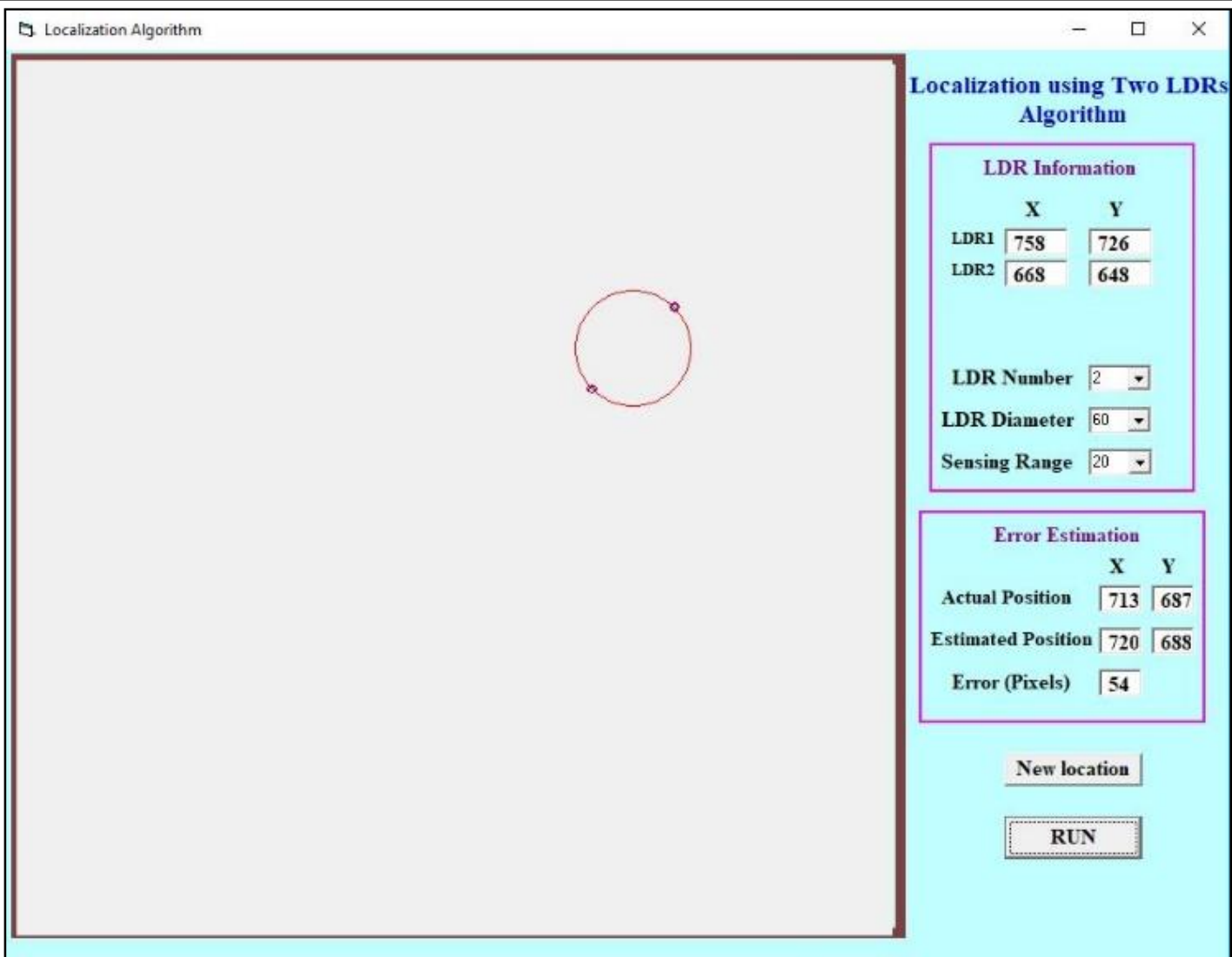

Fig. 18 Simulation for robot localization with a 60 Pixel LDR diameter

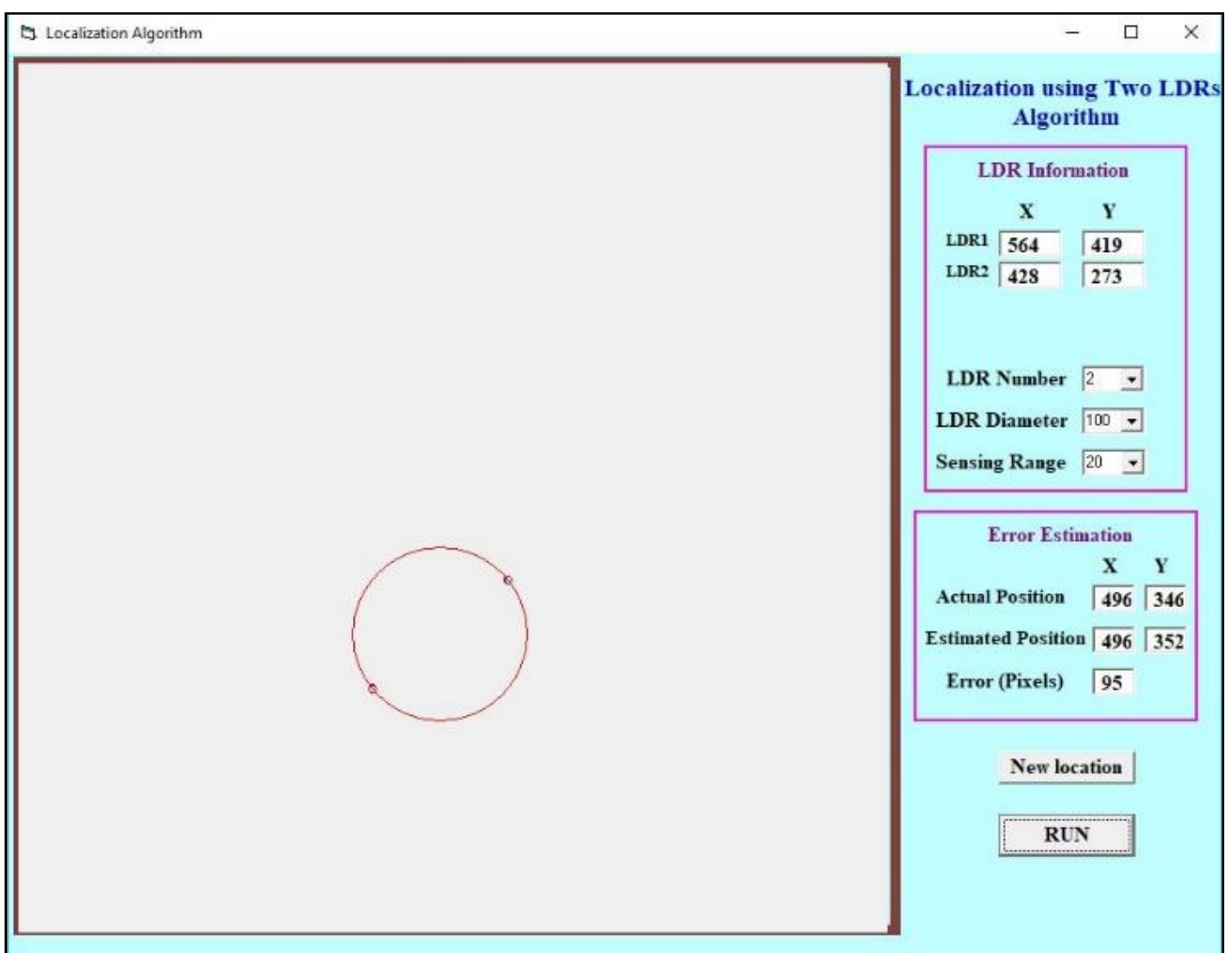

Fig. 19 Simulation for robot localization with 100 Pixel LDR diameter. 


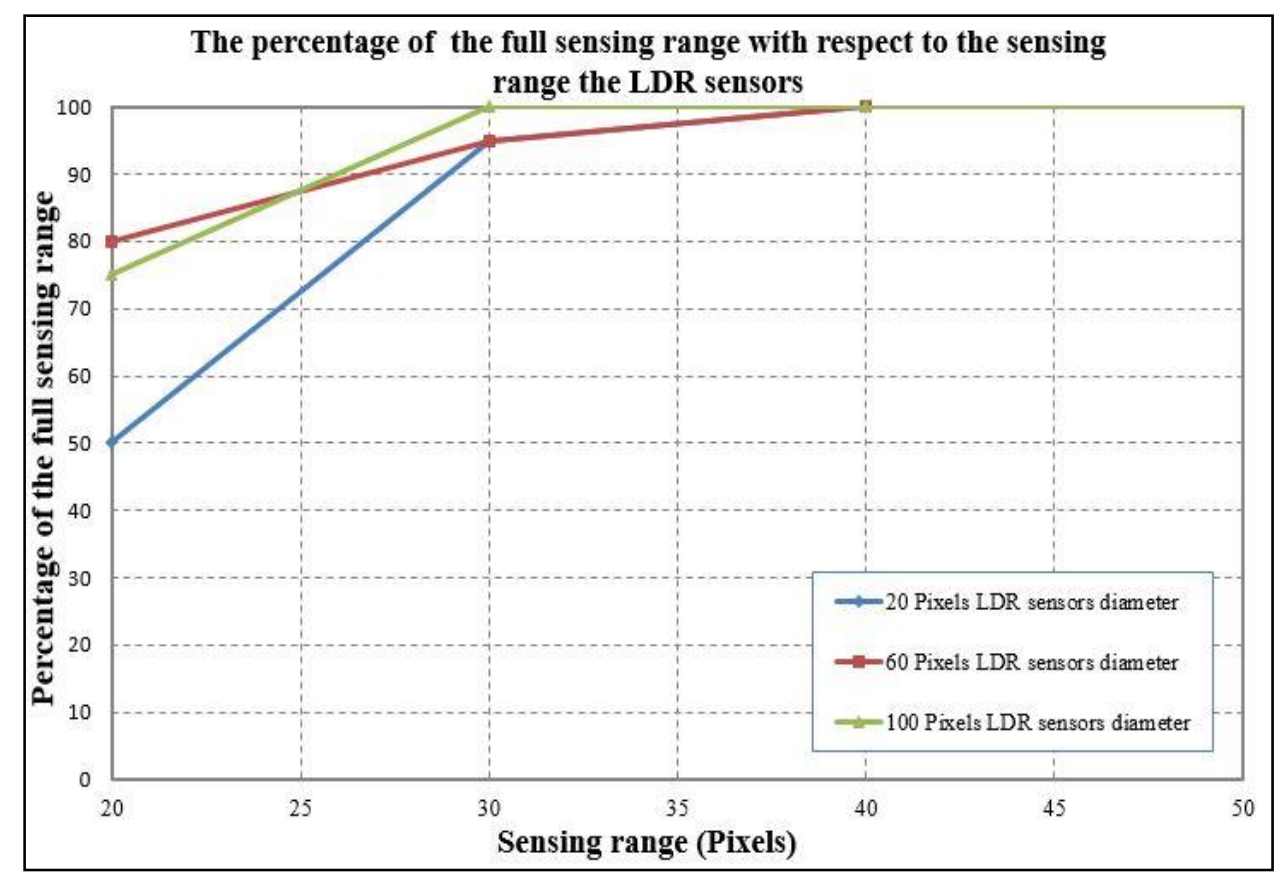

Fig. 20. The relationship between the full sensing range and the LDR sensing range with two LDR robots.

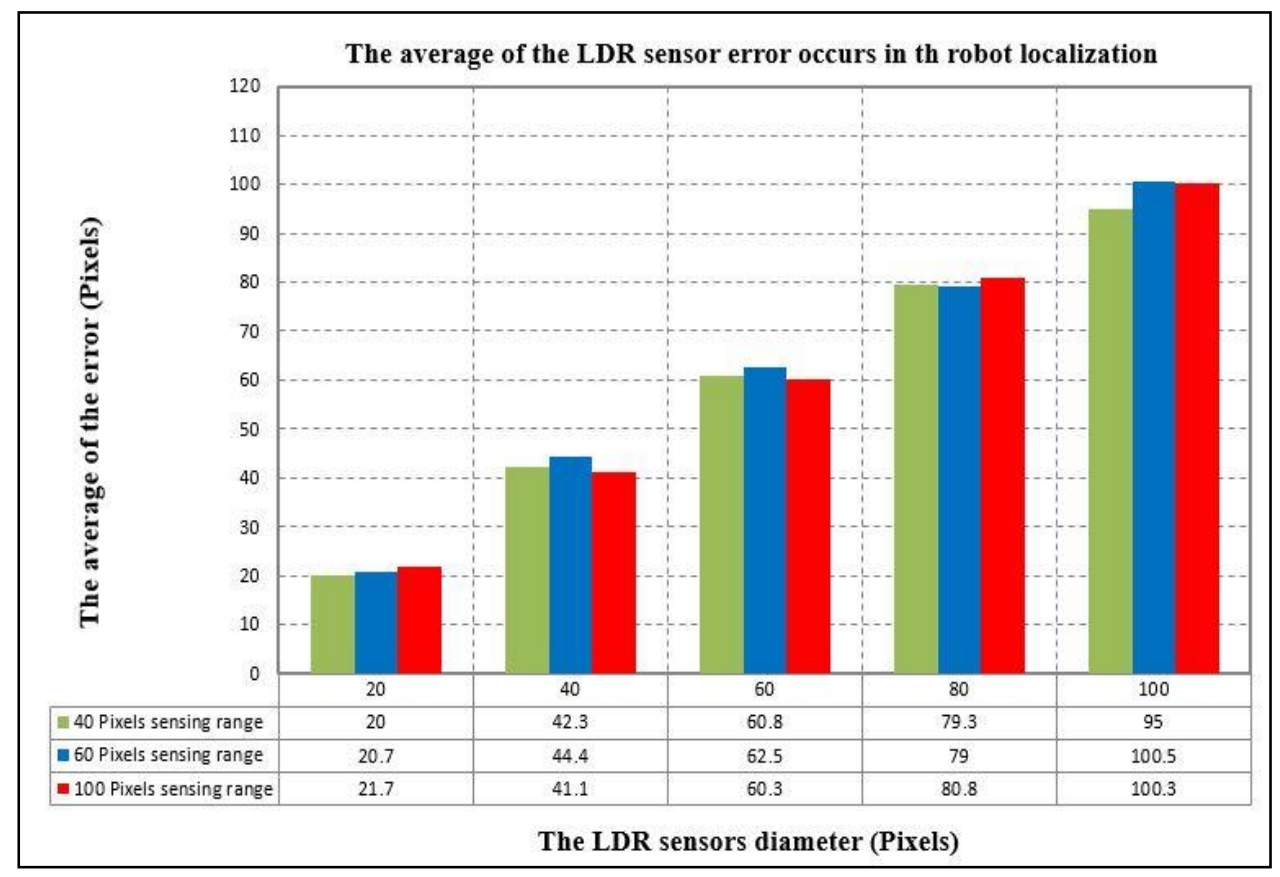

Fig.21. The relationship between the complete sensing range and the LDR sensing range on a robot with two LDR sensors.

\section{CONCLUSION}

This paper introduces an indoor robot localization system. This system solves the problem of localization by using a matrix of $(32 * 32)$ LEDs distributed uniformly in the environment, and a mobile robot equipped with eight LDRs sensors and only two of them(LDRs) are activate the visibility of the robot. According to the proposed system, an efficient equation is proposed to estimate the robot's position by drawing two virtual circles for every two LDR sensors; one of them is true and the other is neglected according to several suggested equations. The midpoint of this circle is assumed to be the robot center. The new 
system is simulated on an environment with LEDs array. The simulation effect of this system displays a good performance in the localization process. The comparisons are tested in the localization process for the robots with different sensing ranges of each LDR (20 to 100 Pixels). This comparison shows that the average error is reduced as the distance between LDRs are also reduced and the best sensing range to obtain a full sensing is at 40 Pixels.

\section{REFERENCES}

[1] R. Tron, J. Thomas, G. Loianno, K. Daniilidis and V. Kumar (2016). A Distributed Optimization Framework for Localization and Formation Control: Applications to Vision-Based Measurements. IEEE Contr. Sys. Mag., 36(4), 22-44.

[2] I. Borg, P. Groenen, Modern Multidimensional Scaling: Theory and Applications, In: Springer Series in Statistics, Springer, 2005.

[3] I. Loevsky, I. Shimshoni, Reliable and efficient landmark-based localization for mobile robots, Robotics and Autonomous Systems 58 (2010) 520_528, www.elsevier.com/locate/robot.

[4] D. Belter and M. Nowicki, Optimization-based legged audiometry and Sensor fusion for legged robot continuous localization, Elsevier, Robotics and

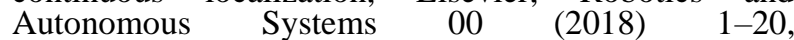
https://doi.org/10.1016/j.robot.2018.10.013.

[5] E. Cole and S. Galerne, Mobile robot localization by multiangulation using set inversion, Elsevier, Robotics and Autonomous Systems 61 (2013) 39-48, www.elsevier.com/locate/robot.

[6] Carmelo DI Franco, Mauro Marinoni, Enrico Bini and Giorgio C. Buttazzo, Dynamic Multidimensional Scaling with anchors and height constraints for indoor localization of mobile nodes, Elsevier, Robotics and

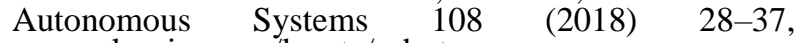
www.elsevier.com/locate/robot.

[7] H. Liu, J. Yang, S. Sidhom, Y. Wang, Y. Chen, F. Yeah, Accurate Wi-Fi based localization for smartphones using peer assistance, IEEE Transactions on Mobile Computing 13 (10) (2014) 2199-2214.

[8] N. B. Priyantha, H. Balakrishnan, E. Demaine, and S. Teller (2003). "Anchor Free Distributed Localization in Sensor Networks “. Tech Report \#892.

[9] Abdulmuttalib T. Rashid, M. Frasca, A, A, Ali, A. Rizzo and L. Foruna (2015) Multi-robot localization and orientation estimation using robotic cluster matching algorithm. Robotics and Autonomous Systems, 63, 108121.

[10] Abdulmuttalib T. Rashid, W. H. Zayer and M. T. Rashid (2018). "Design and Implementation of Locations Matching Algorithm for Multi-Object Recognition and Localization ". Iraqi Journal of Electrical and Electronic Engineering, 14(1), 10-21.

[11] T. Arampatzis, J. Lygeros and S. Manesis (2005). "A Survey of Applications of Wireless Sensors and Wireless Sensor Networks," Intelligent control. Proceedings of the 2005 IEEE International Symposium on, Mediterranean Conference on Control and Automation, $719-724$

[12]I. S. Alfurati , Abdulmuttalib T. Rashid and Alaa AlIbadi (2019). "IR sensors array for robot's localization using $\mathrm{K}$ means clustering algorithm “. International
Journal of Simulation Systems, Science \& Technology, 12.1-12.6.

[13] O. A. Hasan, Abdulmuttalib T. Rashid and R. S. Ali (2016). Centralized approach for multi-node localization and identification. Iraq J. Electrical and Electronic Engineering, 12 (2), 178-187.

[14] O. A. Hasan, Abdulmuttalib T. Rashid and R. S. Ali (2016). "A Hybrid approach for multi-node localization and Identification ". Basrah Journal for Engineering Sciences, 16(2), 11- 20.

[15]H. Liu, H. Darabi, P. Banerjee, and J. Liu (2007). "Survey of Wireless Indoor Positioning Techniques and Systems ". IEEE TRANSACTIONS ON SYSTEMS, MAN, AND CYBERNETICS-PART C: APPLICATIONS AND REVIEWS, 37(6), 1067 - 1080.

[16]I. S. Alfurati and Abdulmuttalib T. Rashid (2018). "Performance Comparison of Three Types of Sensor Matrices for Indoor Multi-Robot Localization ". International Journal of Computer Applications (0975 8887), 181 (26), 22-29.

[17] Y. Gu, A. Lo, and I. Niemegeers, "A survey of indoor positioning systems for wireless personal networks," Communications Surveys \& Tutorials, IEEE, vol. no. 1, pp. 13-32, 2009.

[18] C. Lee, Y. Chang, G. Park, J. Ryu, S.-G. Jeong, S. Park, J. W.Park, "H. C. Lees and K-s. Hong," Indoor Positioning System Based on Incident Angles of Infrared Emitters", The 30th Annular Conference of the IEEE Industrial Electronics Society, November 2 -6, 2004.

[19] X. N. Fernando, S. Krishnan, H. Sun and K. K-Moud," Adaptive denoising at Infrared wireless receivers", The International Society for Optical Engineering . September 2003.

[20]I. S. Alfurati and Abdulmuttalib T. Rashid (2019)," Design and Implementation an Indoor Robot Localization System Using Minimum Bounded Circle Algorithm", in press. 